Document downloaded from:

http://hdl.handle.net/10251/161864

This paper must be cited as:

Gachuz, EJ.; Castillo-Santillán, M.; Juarez-Moreno, C.; Maya Cornejo, J.; Martinez-Richa, A.; Andrio, A.; Compañ Moreno, V.... (2020). Electrical conductivity of an all-natural and biocompatible semi-interpenetrating polymer network containing a deep eutectic solvent. Green Chemistry. 22(17):5785-5797. https://doi.org/10.1039/d0gc02274h

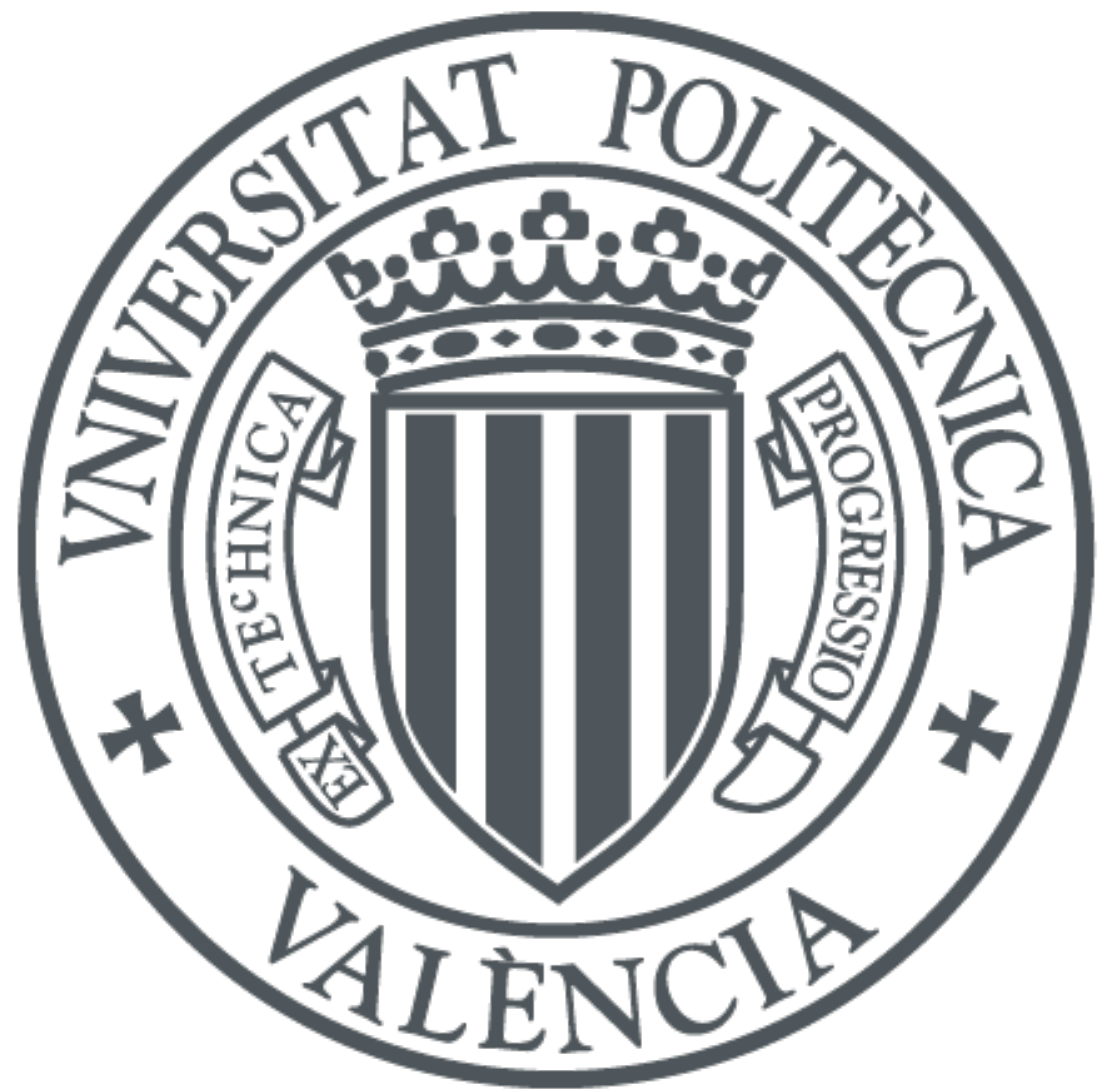

The final publication is available at

https://doi.org/10.1039/d0gc02274h

Copyright The Royal Society of Chemistry

Additional Information 
Received 00th January 20xx, Accepted 00th January 20xx DOI: $10.1039 / x 0 \times x 00000 x$

\title{
Electrical conductivity of all-natural and biocompatible semi- interpenetrated polymer network containing a deep eutectic solvent
}

\author{
Edwin J. Gachuz, ${ }^{a}$ Martín Castillo-Santillán, ${ }^{a}$ Karla Juarez-Moreno, ${ }^{b}$ Jose Maya-Cornejo, ${ }^{a}$ Antonio \\ Martinez-Richa, ${ }^{c}$ Andreu Andrio, ${ }^{d}$ Vicente Compañ, ${ }^{*}$ e Josué D. Mota-Morales*,a
}

\begin{abstract}
A series of semi-interpenetrated polymer networks (semi-IPNs) consisting of crosslinked poly(itaconic acid) in the presence of the polysaccharide inulin were prepared by free-radical polymerization, taking advantage of the deep eutectic systems (DESs) chemistry. Up to $14 \mathrm{wt} \%$ of the polysaccharide inulin readily dissolves in a nonaqueous DES composed of glycerol (Gly) and choline chloride ( $\mathrm{ChCl}$ ). On the other hand, itaconic acid (IA) mixed with $\mathrm{ChCl}$ formed a deep eutectic solvent (DES) monomer, which upon free-radical polymerization in solution aided by multifunctional acrylates, allowed the synthesis of highly crosslinked polymer network. Bringing together both DESs, the DES monomer containing IA and the inert one containing inulin dissolved in it, allowed the synthesis of an all-natural (ca. 96 wt.\% of biobased components, excluding crosslinkers) and biocompatible semi-IPNs. Remarkably, the DESs entrapped in the IPNs served as a stable nonaqueous electrolyte in the range of $20-80^{\circ} \mathrm{C}$ thus exhibiting a typical Arrhenius dependence of conductivity with temperature (apparent activation energy of $18 \mathrm{~kJ} \mathrm{~mol}^{-1}$ ), irrespective of the type of crosslinker used. Following the electrode polarization (EP) analysis based on the Macdonald-Trukhan model, the free-ion diffusivity, the mobility, and the number of charge carrier density of both polymeric networks were calculated. The results show that diffusivity and mobility increase along with temperature in all semi-IPNs with a maximum conductivity of $4.6 \mathrm{mS} \mathrm{cm}^{-1}$ at $65^{\circ} \mathrm{C}$. It is proposed that glycerol-glycerol hydrogen bonding interactions, which are present in pure liquid glycerol, remains in the glycerol-ChCI DES, although the number of interactions of glycerol-glycerol hydrogen bonds slightly diminished/countervailed by additional bonding with the $\mathrm{ChCl}$. In summary, this work furthers the application of the DES chemistry towards the fabrication of greener materials. e.g. natural polymers and biobased feedstocks, with future application in technologies seeking biocompatible conductive gels.
\end{abstract}

\section{Introduction}

The development of new types of flexible ion conducting materials is one of the most important challenges in the field of energy. The emergence of the so-called soft electronics has led to the search of synthetic methods that combine the properties of conductive and semiconductive materials with the flexibility and compositional versatility of polymeric matrixes, e.g. hybrid organic-inorganic composite polymer electrolytes.

Maintaining high ionic conductivities in these soft electronics, together with achieving good mechanical properties even at moderate temperatures without the risk of electrolyte leaking, have been the focus of recent works. Among several

\footnotetext{
a. Centro de Física Aplicada y Tecnología Avanzada (CFATA), Universidad Nacional Autónoma de México, Querétaro, QRO 76230, Mexico

b. Centro de Nanociencias y Nanotecnología (CNyN), Universidad Nacional

Autónoma de México, Ensenada, B.C. 22860, Mexico

c. División de Ciencias Naturales y Exactas, Universidad de Guanajuato, GTO 36050, México.

d. Universitat Jaume I., Castelló 12071, Spain

e. Universitat Politécnica de Valencia, Valencia 46022, Spain.

† Electronic Supplementary Information (ESI) available: FTIR and ${ }^{13} \mathrm{C}$ NMR spectra, and swelling behavior of semi-IPNs. See DOI: 10.1039/x0xx00000x
}

nonaqueous approaches, ionic liquids (ILs) have been considered as attractive electrolytes that improve the performance, safety, long term stability and processing when they take part in the construction of devices such as batteries, fuel cells, supercapacitors, actuators, biosensors among the others. ${ }^{1,2}$

In the quest for sustainable reaction media and solvents for chemical processes, in the last decades, the use of a family of neoteric fluids called deep eutectic solvents (DESs) have emerged for the reactive and non-reactive dissolution of both organic and inorganic solutes, including resilient biomass. ${ }^{3}$ DESs are mixtures of at least one hydrogen-bond donor (HBD) and at least one hydrogen-bond acceptor (HBA) that exhibit a large melting point depression upon mixing, i.e. with a composition corresponding to a eutectic point. ${ }^{4,5}$ Being choline chloride (ChCl) the archetype of HBA in many DESs (specifically type I, II and III DESs as proposed by Abbott et al. ${ }^{5}$ ) these $\mathrm{ChCl}$-based DESs exhibit high ionic conductivities (e.g., fglycerol-ChCl exhibits $7.61 \mathrm{mS} / \mathrm{cm}$ at $20^{\circ} \mathrm{C}$ ) as compared with molecular liquids, which strongly depends, apart from ambient conditions, on the HBA/HBD pair. Mobil charges comes from the chloride anion and quaternary ammonium cation presented in DESs in liquid state. 
This knowledge has been materialized recently with the synthesis of polymeric networks involving DESs in their synthesis. Polymerization of DESs bearing monomers such as (meth)acrylic acids as HBDs or performing the polymerization within a DESs, are the two main approaches reported. ${ }^{6}$ These gel-like materials exhibit remarkable features in addition to ionic conductivity arising from DEs incorporation, such as flexibility, self-healing, scratchable, among others. ${ }^{7-11}$

Likewise, there is a pressing need for sustainable options in the synthesis of new polymeric materials that help reduce the current dependence on petroleum-based polymers. In response, the last years have seen a tremendous growth in the use of biomass as building blocks and raw materials due to its potential biodegradability and large-scale availability. Specifically, among the naturally occurring molecules that biomass encompasses, polysaccharides have found myriad of applications as building blocks in the pharmaceutical and biomedical fields. However, polysaccharides and biobased polymers playing a key role in the developing of advanced functional materials such as photonic materials, ionogels, and actuators, are gaining interest. ${ }^{12,13}$ One of the main challenges for valorization of biomass relies on its solubilization for their subsequent chemical modification and integration into engineered polymers and functional materials.

Herein, a semi-IPN consisting of crosslinked poly(itaconic acid) (PIA) and the polysaccharide inulin was prepared by using different facets of the $\mathrm{ChCl}$-based DESs: i) IA was first transformed into a DES monomer by mixing with $\mathrm{ChCl}(1: 1$ molar ratio, $57^{\circ} \mathrm{C}$ m.p.), and ii) a high concentration of polysaccharide inulin (14 wt\%) was dissolved in a non-reactive DES, namely $\mathrm{Gly}-\mathrm{ChCl}, 2: 1$ molar ratio, $-40^{\circ} \mathrm{C}$ m.p. Bringing together both DESs, IA-ChCl and glycerol-ChCl (the latter containing inulin dissolved in it), and polymerizing the IA by free-radical polymerization, yielded a biocompatible (tested with human fibroblasts and murine macrophages) semi-IPN composed of a biobased monomer (itaconic acid) and a polysaccharide (inulin). Thanks to the ionic nature of the DESs entrapped in the semi-IPNs (coming from the $\mathrm{ChCl}$ ), they served as a stable nonaqueous electrolytes in the range of $20-80^{\circ} \mathrm{C}$. The effect of temperature on the conductivity, diffusivity and free charge density in the all-natural and biocompatible semi-IPN were then studied in detail. The electrode polarization (EP) model introduced by Klein et al. ${ }^{14}$ was followed to determine the free-charge density, the mobility and the diffusion coefficient, using the analysis of the frequency dependence of complex dielectric loss $\varepsilon^{\prime \prime}$ and loss $\tan \delta$. A discussion with respect to the formalism used by Bandara et al. ${ }^{15}$ was then stablished.

\section{Results and discussion}

\section{IPN preparation.}

Solubilization of the polysaccharide inulin has been reported in reactive solvents including ILs based on 1-butyl-3-methyl imidazolium and 1-ethyl-3-methylimidazolium cations, ${ }^{16,17}$ and deep eutectic solvents (DESs) composed of fructose, and citric and oxalic acids, all mixed with $\mathrm{ChCl}^{18,19}$ In these works, not only inulin solubilization was studied, but also its depolymerization and transformation into hydroxymethylfurfural building blocks in one pot.

In this work the first step towards the preparation of inulin based semi-IPNs consisted in the selection of a suitable nonreactive solvent for the polysaccharide inulin. Several DESs based upon mixtures of hydrogen bond donors (HBDs) and $\mathrm{ChCl}$ (as the HBA) were tested, including citric acid (anhydrous and hydrated), glycerol and acetamide, which were mixed with $\mathrm{ChCl}$ with different molar ratio. Gly-ChCl DES (2:1 molar ratio, respectively) was selected because its moderate viscosity (376 $\mathrm{CP}$ at $25^{\circ} \mathrm{C}$ ) and its capability to stablish hydrogen bonding with saccharides ${ }^{20}$ and polymers, ${ }^{21-23}$ including polysaccharides. ${ }^{24,25}$ As result, homogenous dissolution of up to $14 \mathrm{wt} \%$ of the polysaccharide inulin was possible at room temperature without chemical derivatization, and without gel formation. ${ }^{26}$ To verify the chemical integrity of inulin during and after dissolution, fully precipitated and recovered inulin from the Gly$\mathrm{ChCl}$ DES was studied by FTIR spectroscopy. This was done by addition of water as antisolvent of inulin (at room temperature) that, conversely, was a good solvent for glycerol and $\mathrm{ChCl}$. No appearance of new chemical bonds was detected in the recovered inulin, having the same characteristics than the pristine one (Supporting Information, Figure S1). DESs are known to dissolve large amounts of polysaccharides, but this is a remarkable result, taking into account that solubility of inulin in water is ca. $1.2 \% \mathrm{w} / \mathrm{v}$ at $50^{\circ} \mathrm{C}^{26}$ that is, 10 -fold less than that in $\mathrm{Gly}-\mathrm{ChCl} \mathrm{DES}$ at room temperature.

Together with the capability of the DES media to stablish hydrogen bonding with polymers, temperature played an important role in achieving the homogenous dissolution of a large amount of inulin. As abovementioned, inulin has been used as a natural source of furfural building block via its depolymerization, which occurs above $100^{\circ} \mathrm{C}$ in DESs composed of fructose, and citric and oxalic acids as $\mathrm{HBDs}$, and $\mathrm{ChCl}$ as $\mathrm{HBA}$. Similarly, direct dissolution of inulin at temperatures above $70^{\circ} \mathrm{C}$ in IA-ChCl (1:1 molar ratio), Gly-ChCl (2:1 molar ratio), and acetamide- $\mathrm{ChCl}$ (2:1 molar ratio) produced brown solutions within minutes as result of inulin degradation. In the case of citric acid- $\mathrm{ChCl}$, additionally, the high viscosity difficulted the homogeneous dissolution of inulin.

In a second step, it was verified the feasibility of the free-radical polymerization of IA forming DES with $\mathrm{ChCl}$ (i.e., DES monomer) in solution, using of Gly-ChCl DES as inert reaction media (Figure 1). It has been demonstrated that acrylates playing the role of hydrogen bond donors (HBD) can be transformed into DES monomer by mixing with $\mathrm{ChCl}$ with the appropriate molar ratio. Examples include acrylic acid, ${ }^{6}$ methacrylic acid, ${ }^{27}$ acrylamide and IA (IA-ChCl DES, $\left.\mathrm{T}_{\mathrm{m}}=57^{\circ} \mathrm{C}\right) .{ }^{28}$ All these DESs monomers can be subjected to free-radical polymerization in bulk. Interestingly, Bednarz et al. reported that free-radical polymerization of $\mathrm{IA}-\mathrm{ChCl} \mathrm{DES}$ monomer in bulk, crosslinked with a bifunctional acrylate, e.g. bis-acrylamide (BAA, 5 mol\% to monomer), and initiated by ammonium persulfate (APS, $5 \mathrm{~mol} \%$ to monomer), proceeded faster and at lower temperature than in water. ${ }^{29}$ Moreover, the degree of crosslinking was higher in 
the case of DES, thus pointing to a catalytic effect of choline cation. Here, "in bulk" means that the polymerization was performed in the neat DES monomer without extra solvent; although the IA monomer playing the role of HBD is "diluted" in the non-polymerizable counterpart of the DES (HBA, in this case the $\mathrm{ChCl}$ ) and hence is not bulk in the context of conventional free-radical polymerizations.<smiles></smiles>

Figure 1. Scheme of free-radical polymerization of IA-ChCl DES in solution. (Entries 3 and 4 in Table 1 ).

Similar to previous reports on the free-radical polymerization of acrylamide in Gly-ChCl DES, ${ }^{30}$ the free-radical polymerization of $\mathrm{IA}-\mathrm{ChCl}$ DES monomer proceeded in solution in $\mathrm{Gly}-\mathrm{ChCl}$ DES with high conversions (75\%). Free standing polymer monoliths resulted from washing out the DESs inert components, i.e. Gly and $\mathrm{ChCl}$, and unreacted monomers, due to crosslinking of PIA with multifunctional acrylates, namely BAA or pentaerythritol triacrylate (PETA) (Entries 3 and 4 in Table 1). The use of two types of crosslinkers, bi- and trifunctional, aimed to study the effect of the degree of crosslinking on the swelling behavior of the semi-IPN as well as on the conductivity of DES-containing semi-IPNs; in the latter case, differences are expected due to formation of denser structure supporting the liquid electrolyte upon polymerization.

Bringing together both DESs: i) the neat DES monomer composed of IA (IA-ChCl DES), and ii) the inert one (Gly-ChCl) containing a high concentration of inulin dissolved in it (14 wt\%), allowed the synthesis of an all-natural and biocompatible semi-IPN upon free-radical polymerization of IA aided by BAA or PETA multifunctional acrylates (Figure 2). The compositions of the semi-IPNs studied are listed in Table 1.

Table 1. Feed composition of semi-IPNs and crosslinked PIA.

\begin{tabular}{|c|c|c|c|c|c|c|}
\hline \multirow{2}{*}{ Entry } & $\begin{array}{c}\text { IA-ChCl } \\
\text { DES } \\
\mathrm{mmol}: \\
\mathrm{mmol}\end{array}$ & $\begin{array}{c}\text { Gly-ChCl } \\
\text { DES } \\
\mathrm{mmol}: \\
\mathrm{mmol}\end{array}$ & $\begin{array}{c}\text { Inulin } \\
\mathrm{mg}\end{array}$ & $\begin{array}{c}\text { BAA } \\
\text { mmol }\end{array}$ & $\begin{array}{c}\text { PETA } \\
\mathrm{mmol}\end{array}$ & $\begin{array}{c}\text { APS } \\
\mathrm{mmol}\end{array}$ \\
\hline 1 & $6.6: 6.6$ & & & 0.33 & & 0.307 \\
\hline 2 & $6.6: 6.6$ & & & & 0.33 & 0.323 \\
\hline 3 & $6.6: 6.6$ & $7.1: 3.55$ & & 0.33 & & 0.307 \\
\hline 4 & $6.6: 6.6$ & $7.1: 3.55$ & & & 0.33 & 0.323 \\
\hline 5 & $6.6: 6.6$ & $7.1: 3.55$ & 187 & 0.33 & & 0.307 \\
\hline 6 & $6.6: 6.6$ & $7.1: 3.55$ & 187 & & 0.33 & 0.323 \\
\hline
\end{tabular}

\section{Characterization of IPN}

Incorporation of inulin to hydrogels has been reported before. In these reports, chemical derivatization of inulin was used as an strategy to graft polymerizable moieties, e.g. acrylate functionalities. ${ }^{31-33}$ However, chemical modification of inulin to render crosslinked networks might compromise their physicochemical properties and biodegradability to function as, for example, hydrogels for colonic or oral drug delivery systems. ${ }^{34}$ Here, taking advantage of the nonaqueous environment provided by the DES, it is reported the direct incorporation of inulin to an all-natural semi-IPN, i.e. without chemical derivatization.

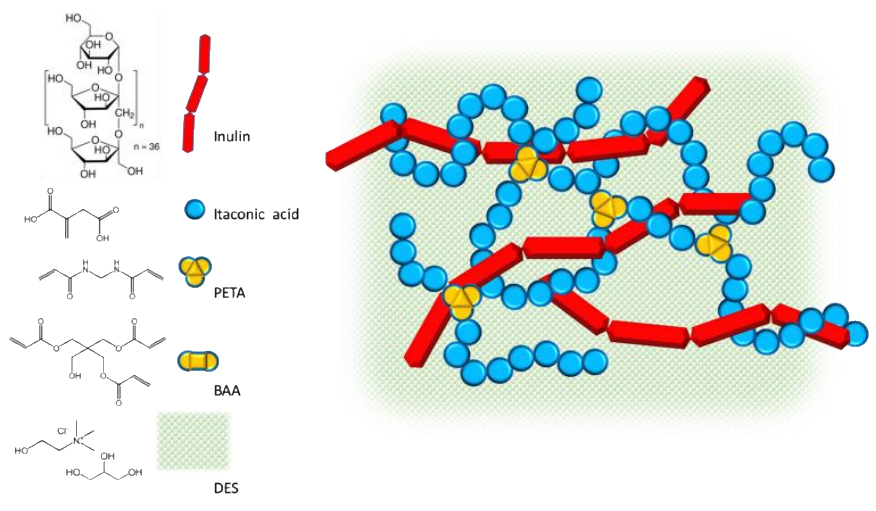

Figure 2. General scheme of formation of semi-IPN by the freeradical polymerization of IA-ChCl DES monomer and crosslinking in the presence of inulin dissolved in Gly-ChCl DES.

To avoid thermal degradation, inulin was first dissolved in Gly$\mathrm{ChCl}$ DES at room temperature and subsequently, upon mixture, the free-radical polymerization of IA-based DES monomer in the inulin solution was carried out at $60^{\circ} \mathrm{C}$. Attenuated Total Reflectance-Fourier Transformed Infrared (ATR-FTIR) spectrum of Gly-ChCl DES containing inulin didn't show noticeable shifts as compared with neat DES (Supporting Information Fig. S2), despite containing a high concentration of the polyfructane without gel formation. Thus, a possible ternary DES formation cannot be ruled out. ${ }^{35,36}$

Formation of the semi-IPNs comprising poly(IA-co-BAA)/inulin PIA-BAA/inulin- and poly(IA-co-PETA)/inulin -PIA-PETA/inulinwere proved by ATR-FTIR and solid state ${ }^{13} \mathrm{C}$ NMR upon extraction of the inert DES components (glycerol and choline chloride), and unreacted monomers with water. The spectra of the semi-IPN in Figure $\mathbf{3 A}$ show the typical signals of the carbonyls groups characteristic of pure PIA, as previously reported; ${ }^{29,37}$ as well as the bands related with $\mathrm{OH}$ and $\mathrm{COC}$ typical of inulin. ${ }^{38}$ Characteristic bands of PIA include those due to $\mathrm{C}=\mathrm{O}$ stretching at $1720 \mathrm{~cm}^{-1}, \mathrm{CH}_{2}$ bending at $1430 \mathrm{~cm}^{-1}, \mathrm{O}-\mathrm{H}$ bending at $1400 \mathrm{~cm}^{-1}$, and $\mathrm{C}-\mathrm{O}$ stretching at $1200 \mathrm{~cm}^{-1}$. On the other hand, inulin shows the typical broad band associated with hydroxyl groups between $3100-3600 \mathrm{~cm}^{-1}$, aliphatic $\mathrm{CH}_{2}$ at 2920 $\mathrm{cm}^{-1}$, and those of the fructose ring at 950 and $1150 \mathrm{~cm}^{-1}$; the latter are overlapped with the signals of PIA. Only the FTIR spectra of PIA-PETA/inulin is shown; no differences were observed between PETA and BAA due to their low concentration (Supplementary Info. Fig. S3). Two main features in the semi-IPN spectra reflect the presence of inulin, the broad 
band of hydroxyls at $3100-3600 \mathrm{~cm}^{-1}$ and the band related with glycosidic bonding C-O-C at $1030 \mathrm{~cm}^{-1}$ (Figure 3A). ${ }^{38}$

Carbon resonance profiles in the solid state are sensitive to distributions in molecular arrangement, thus, to further demonstrate the successful formation of the semi-IPN, solid state ${ }^{13} \mathrm{C}$ NMR of the different materials synthesized were carried out. In the spectra of Figure $3 B$ it is compared the signals of pure inulin with the semi-IPN, that is, the crosslinked PIA polymerized in the presence of inulin. Further comparison with crosslinked PIA without inulin is shown in the Supplementary Info. Fig. S4. The carbonyls of IA monomeric unit are found at 173.5 and $178 \mathrm{ppm}$, although given the crosslinked nature of the polymer they appear broad and overlapped. Quaternary and methylene carbons are found at 46, 43 and 35 ppm, respectively. It is worthy to note that the sharp signal at $54 \mathrm{ppm}$ has been associated to choline grafting to the PIA backbone via ester formation during IA free-radical polymerization. ${ }^{29}$

Inulin exhibits the typical peaks associated with polysaccharides in the range of 55 to $110 \mathrm{ppm}$. Specifically, the peak at $104 \mathrm{ppm}$ belong to anomeric $C(2)$ carbon, 81-83 ppm to $C(5), 74-78 \mathrm{ppm}$ to $C(3)$ and $C(4)$ carbons. Peaks located from 64 to $58 \mathrm{ppm}$ are assigned to $C(6)$ and $C(1)$ respectively (Inset Figure $3 B$ ). All these signals belong to fructose units. As with FTIR, no significant differences due to the use of two different crosslinkers, BAA and PETA, were observed. However, by comparing the signals of pure inulin with the corresponding carbons of the polysaccharide in the semi-IPN, there is a noticeable broadening of the peaks and a shift of ca. $10 \mathrm{ppm}$ associated to the entanglement of inulin chains in the crosslinked network of PIA formed in situ.
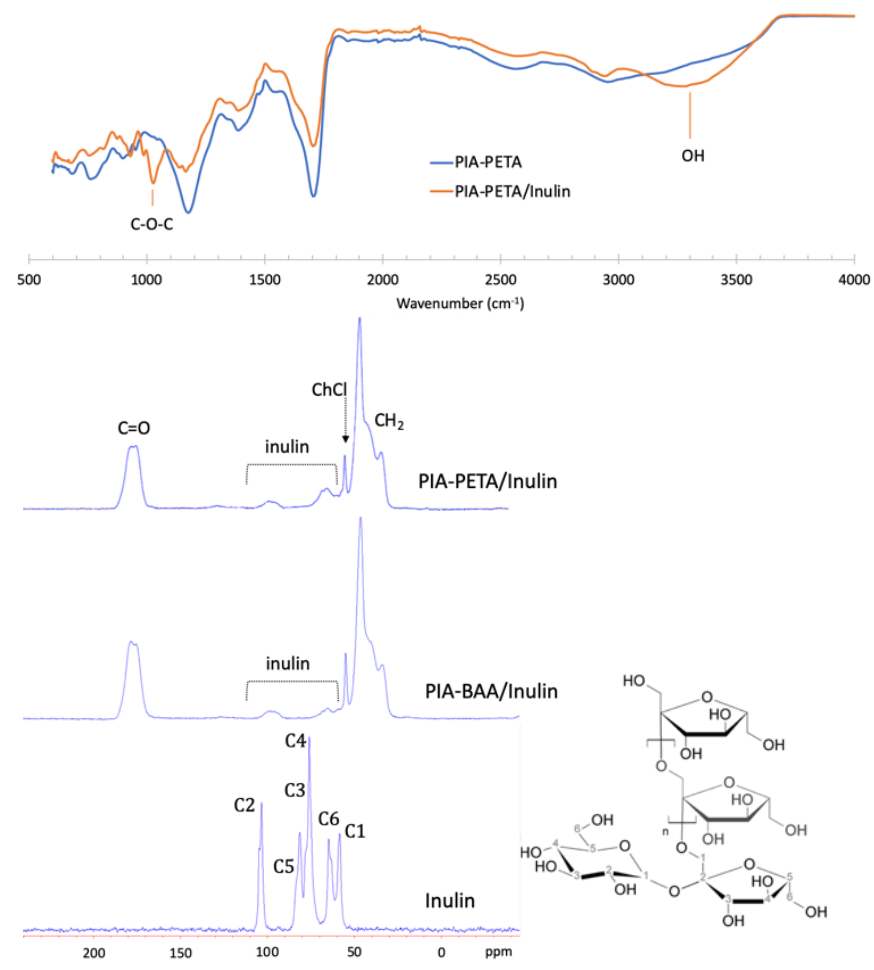

Figure 3. FTIR and $13 C$ NMR spectra of semi-IPNs.
Similarly, peaks of cellulose analyzed by ${ }^{13} \mathrm{C}$ NMR exhibited broadening and important shifts as result of its association with glucans. ${ }^{39}$ Glucans incorporated into the highly ordered structure of cellulose, disrupted the formation of $\mathrm{H}$-bond, causing a significant loss in crystallinity. Considering the hydrogen bond formation ability of pendant carboxylic acids in PIA and the abundant hydroxyl moieties of inulin, their intermolecular association is expected, mainly in a water-free environment that circumvent ionization of carboxyl groups.

To get insights into the formation of semi-IPN between the inulin and the crosslinked PIA, solid PIA previously synthesized in $\mathrm{Gly}-\mathrm{ChCl}$ was mixed by grinding with solid inulin to form a mixture. On the other hand, crosslinked PIA was swelled with a solution of inulin previously dissolved in water and the resulted hydrogel was subjected to freeze-drying. (Supplementary Info. Fig. S5) ${ }^{13} \mathrm{C}$ NMR of these mixtures exhibited, in addition to peaks associated with PIA, the well-defined peaks of inulin, in contrast with the broad and upfield shifted peaks of inulin in the semi-IPNs. This confirms that the association between both polymers, inulin and PIA, in the form of a semi-IPN only occurred by the in situ free-radical polymerization of IA in the presence of inulin.

\section{Swelling behavior}

Hydrophilic polymer networks uptake large quantities of water to form hydrogels. Depending on the crosslinking degree and the presence of ionizable groups (e.g. carboxylic acid) in the network, hydrogels swelling can be modulated. Given the polyelectrolyte character of polyitaconic acid, ${ }^{28}$ and the abundance of hydroxyl groups in inulin, ${ }^{26}$ they are known to form hydrogels. The swelling behavior of the semi-IPNs upon removal of the DESs was studied at different $\mathrm{pH}$ in water. All semi-IPNs exhibited a pH-responsive swelling behavior due to the establishment of hydrogen bonding between the hydroxyl group in the inulin and carboxylic acid groups in PIA within the network (Supplementary Info. Fig. S5), as observed before for poly(acrylic acid)/polyvinyl alcohol complexes. ${ }^{40}$ Accordingly, higher $\mathrm{pH}$ (e.g. 10) promote the ionization of carboxylic pendant groups in PIA and hence a higher swelling degree, while low $\mathrm{pH}$ (e.g. 4) has the opposite effect.

Different degree of crosslinking in PIA, that is, by using a bifunctional (BAA) and trifunctional (PETA) crosslinkers, also affects the swelling behavior of the semi-IPNs, being higher the swelling in the case of BAA than in PETA, as expected. ${ }^{41}$ It is important to note that incorporation of inulin to the crosslinked PIA network cause the swelling of the semi-IPN being higher without losing the original shape of the dry semi-IPNs. This effect is associated to formation of a semi-IPN. ${ }^{42}$ On the other hand, the swelling of the semi-IPNs was compared with semiIPN composed of poly(acrylic acid) and poly(methacrylic acid), also containing inulin (prepared by the same protocol as the PIA-containing semi-IPNs). In all cases the swelling of PIA-based semi-IPN surpasses that of poly(acrylic acid) and poly(methacrylic acid) ones, ${ }^{6}$ due to the higher content of carboxylic groups. 


\section{IPN Biocompatibility}

The development of conductive gel-like materials that function while in close contact with biological surfaces without eliciting cytotoxic effects is a current challenge; doing so within a sustainable framework and with biobased components adds additional challenges. For this part of the investigation, the cytotoxic effect of PIA-PETA/inulin were tested in vitro upon removal of the electrolytes, i.e. Gly and $\mathrm{ChCl} D E S$, on human fibroblast HFF1 cell line (ATTC SCRC-1041) and murine macrophages RAW 264.7 (TIB-71). PIA-BAA/inulin was not included in the experiments as unreacted bis-acrylamide monomer is related to cytotoxic effects.
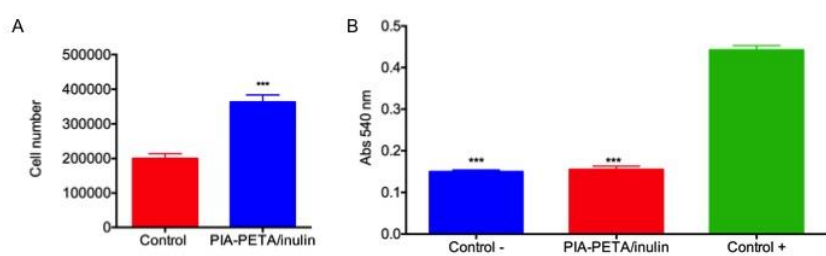

Figure 4. A) biocompatibility assays of PIA-PETA/inulin in human fibroblasts HFF1 after $24 \mathrm{H}$. B) Production of nitrites by RAW 264.7 murine macrophages culture on PIA-PETA/inulin at $24 \mathrm{H}$.

Upon cell culture, the PIA-PETA/inulin semi-IPN samples were equilibrated $(24 \mathrm{H})$ in cell culture $(\mathrm{pH}=7.4)$ media following the results of their swelling behavior. Figure 4 depicts the results of biocompatibility assays of semi-IPNs in human fibroblasts HFF1 (A). It shows the number of HFF1 cells that grew on the surface of the swollen PIA-PETA/Inulin semi-IPNs after $48 \mathrm{H}$ of culture. In comparison with cells growing only in cell culture medium (control), it is important to note that cell population doubles its number after $48 \mathrm{H}$ of incubation on the inulin semi-IPNs. This clearly indicates that the PIA-PETA/Inulin semi-IPN are not only biocompatible but remarkably, it also induces the duplication of fibroblasts population. This behavior opens the path for using the semi-IPNs as novel cell scaffold in tissue engineering. For example, Micic et al, showed that the incorporation of itaconic acid to a polyacrylate improved the biocompatibility in subconjunctival tissue cell culture. ${ }^{43}$ On the other hand, inulin has shown absence of toxicity (including its degradation products), biocompatibility, and nutritional properties. ${ }^{44}$ Due to the fact that the inulin scaffold is based on a polysaccharide, it is crucial to evaluate whether it triggers inflammatory responses. Thus, the effect of inulin semi-IPNs was evaluated on the production of nitrites by RAW 264.7 murine macrophages. Measuring of nitrites is an indirect valid approach to elucidate whether macrophages produce nitric oxide, which is involved in the inflammatory response elicited by this type of immune cell. In Figure 4 (B) the nitrites production is depicted by the absorbance of Griess reagent at $540 \mathrm{~nm}$. As observed, the absorbance of the nitrites production of macrophages exposed to inulin-containing semi-IPNs is comparable to that obtained by macrophages cultivated alone in cell culture media (C-). However, both values are statistically lower than those obtained by inducing nitric oxide production with lipopolysaccharides (LPS) in macrophages $(\mathrm{C}+)$. Therefore, the
PIA-PETA/Inulin semi-IPNs scaffolds did not induce cytotoxic and in vitro inflammatory responses. Thus, the semi-IPN composed of biobased components, inulin and a crosslinked PIA, pose an excellent biocompatibility evidenced by the low to null cytotoxicity, a remarkable result considering the low tolerance of macrophages against foreign materials.

\section{Conductivity and charge transport}

Conductivity and charge transport occurring in DESs has been the subject of recent works. ${ }^{45-47}$ In general, for ChCl-based DESs, it is well stablished that conductivity depends on the type of HBA as well as on the temperature, with values of conductivity for neat DES spanning from $10^{-3}$ to $7.6 \mathrm{mS} \mathrm{cm}^{-1} .{ }^{48} \mathrm{In}$ this work, the Gly-ChCl DES served as not only the media were inulin was dissolved but, upon free-radical polymerization of IA, it was also the nonaqueous electrolyte entrapped into the semi-IPN. Hereafter these semi-IPNs containing Gly-ChCl DES will be simply referred as semi-IPN followed by the corresponding composition.
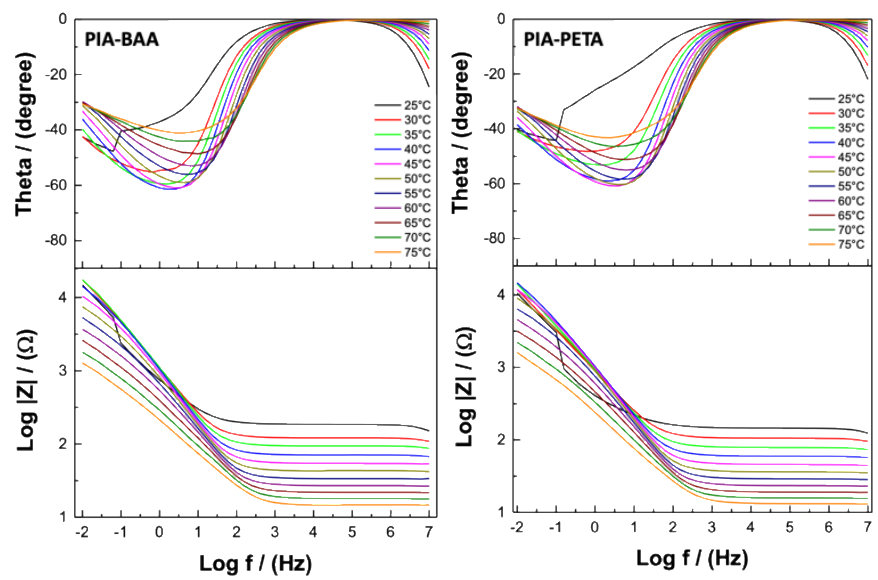

Figure 5. Bode diagram for the a) PIA-BAA/Inulin and b) PIAPETA/Inulin materials at different temperatures, both containing Gly-ChCl DES.

The Bode diagrams for the semi-IPNs (PIA-BAA/Inulin and PIA$P E T A /$ Inulin), containing the DES entrapped are given in Figure 5. These figures show the variation of the conductivity versus frequency for PIA-BAA/Inulin and PIA-PETA/Inulin, at different temperature. A close inspection of Figure 5 shows that both samples have a constant value of the real part of the conductivity when the phase angle is zero (i.e. the impedance of the sample is constant). It is also observed a transition zone between $10^{3}$ to $10^{1} \mathrm{~Hz}$, due to electrode polarization being completely build. Finally, it appears a zone where the conductivity tends to zero when the frequency decreases. The first process is directly related with the resistance/stability of the material and the second process is related with the diffusion (mass transfer) due to the mobility of the cation choline, the anion chloride and glycerol that were entrapped into the polymer matrix (semi-IPN).

The values of the conductivity have been obtained from the plateau at the frequency where the phase angle was zero. For 
example, the conductivity values obtained at $25^{\circ} \mathrm{C}$ for the PIA$\mathrm{BAA} /$ Inulin and PIA-PETA/Inulin samples were quite similar because the maximum values were around $3.4 \times 10^{-4}$ and $4.2 \times 10^{-}$ ${ }^{4} \mathrm{~S} \mathrm{~cm}^{-1}$, and at $65^{\circ} \mathrm{C}$ the values were $2.8 \times 10^{-3}$ and $3.2 \times 10^{-3} \mathrm{~S} \mathrm{~cm}^{-}$ 1 , respectively. The frequencies range for the highest values of the conductivity were $10^{7}$ to $10^{3} \mathrm{~Hz}$ in both cases. Furthermore, the conductivity in those frequencies ranges is constant and this behavior is related with a good stability of the polymer matrix with the trapped nonaqueous electrolyte, i.e. the DES.
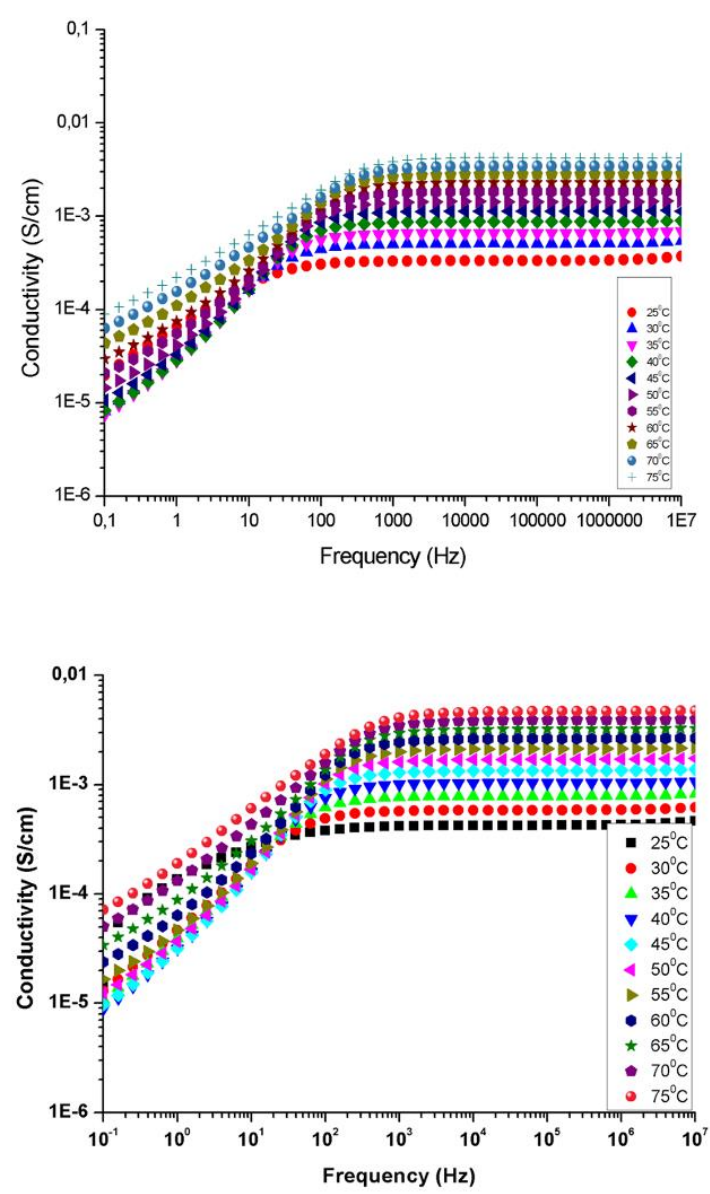

Figure 6. Conductivity versus frequency at different temperatures for the sample: Top) PIA-BAA, and bottom) PIA-PETA.

In the same way, there was an expected mass diffusion phenomenon in the frequency ranges between $10^{1}$ to $10^{-1} \mathrm{~Hz}$ due to the sample's exposition to polarization, promoting the mass transport of ions in the DES electrolyte within the semiIPN. When the temperature of the analysis increases, both PIABAA/Inulin and PIA-PETA/Inulin exhibited a good stability because the values of the real part of the conductivity, $\sigma_{d c}$, were constant until the process related with the mass diffusion appeared.

A close inspection of the Figure 6 shows that conductivity values of PIA-BAA and PIA-PETA are quite similar to those obtained for $\mathrm{ChCl}$-glycerol DES which values were compress between 0.106 and $1.047 \mathrm{mS} / \mathrm{cm}$ at $20 \circ \mathrm{C}$. On the other hand, our values are the same order of magnitude that $\mathrm{ChCl}$-urea and $\mathrm{ChCl}$-Urea-Gly depending of the of glycerol content. For example for $x=0.1$ to 0.9 mole ratio of glycerol in $\mathrm{ChCl} /$ urea/glycerol $(1: 2: \mathrm{x})$, the conductivity varies between 0.822 to $0.911 \mathrm{mS} / \mathrm{cm}$, respectively, ${ }^{49}$. On the other hand, the conductivity values doubled those of $\mathrm{ChCl}$-urea at $40^{\circ} \mathrm{C} .{ }^{50} \mathrm{~A}$ comparison with some solid state electrolyte such as composites of polyethylene oxide (PEO) with different ILs shown that the semi-IPNs ionic gels have values higher than the range of values found for PEO/ILs which ranged between 0.01 to $2 \mathrm{mS} / \mathrm{cm}^{51-53}$

Figure 7 shows the temperature dependence of the conductivity, where a typical Arrhenius behavior is clearly observed. The apparent activation energy was obtained from the slope of the linear fitting and corresponds to $18.3 \pm 0.3$ and $17.8 \pm 0.3 \mathrm{~kJ} \mathrm{~mol}^{-1}$ for the PIA-BAA/Inulin and PIA-PETA/Inulin samples, respectively. This energy can be related with the minimum energy that the polymer network and the DES electrolyte required to begin the ionic migration of the $\mathrm{Ch}^{+}$and $\mathrm{Cl}^{-}$ions and it is directly related with the high conductivity that the polymer composite exhibits (semi-IPN + deep eutectic solvent).

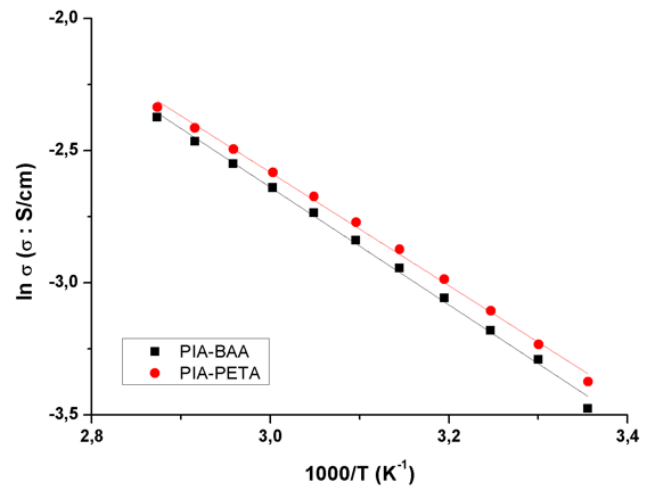

Figure 7. Arrhenius plot of conductivity for PIA-BAA/Inulin (ם) and PIA-PETA/Inulin (•).

The phenomenon of dipolar relaxation in polymers containing electrolytes has not been studied in detail in the high frequency region owing to an overlapping with conductive effects arising from the presence of free ions in the polymeric matrix.

To solve this problem many researchers aimed to understand better this phenomenon by means of models based on the Nernst-Planck equations of electrodiffusion. ${ }^{54}$ Others have adopted empirical methods based on the analysis of electrode polarization (EP) effects. ${ }^{55-57}$ These models are based on the fact that, in the steady state, a sample sandwiched between two electrodes and under the action of an alternating electric field, using blocking electrodes as boundary conditions, behaves as a macroscopic dipole. In such a scenario, it is obtained an expression relating the complex dielectric constant to 
constitutive parameters of the material sandwiched, where a diffusion relaxation time is defined as:

$\tau=\frac{\varepsilon}{\sigma_{d c}}$

where $\varepsilon$ is the polymer dielectric constant $\varepsilon=\varepsilon_{\infty} \varepsilon_{0}$, being $\varepsilon_{\infty}$ the dielectric permittivity at high frequencies and $\varepsilon_{0}$ the vacuum permittivity, and $\sigma_{\mathrm{dc}}$ the sample direct current conductivity. The simpler case is represented by a Debye relaxation as: ${ }^{58}$

$\varepsilon_{E P}^{*}=\varepsilon_{\infty}+\frac{\Delta \varepsilon_{E P}}{1+\left(j \omega \tau_{E P}\right)}$

Where, $\Delta \varepsilon_{E P}=\varepsilon_{E P}-\varepsilon_{\infty}, \omega$ is the angular frequency, $\varepsilon_{E P}$ the permittivity when the electrode polarization is completely build, and $\tau_{E P}$ the electrode polarization relaxation time.

Defining $M=L / 2 L_{D}$, being $L_{D}$ the Debye length, which in turn is defined as $L_{D}=\left(\varepsilon_{S} \varepsilon_{0} k T / q^{2} n\right)^{1 / 2}$, where $k$ is the Boltzmann constant, $T$ the absolute temperature, $q$ is the ion charge and $n$ the mobile charge concentration, and $L$ the sample thickness (i.e. electrode separation when the sample is sandwiched to made the measurements).

The relaxation time of the sample defined as in Eq. (1) when the electrode polarization (EP) is completely build-up, it can be given as, 58,59

$\tau_{E P}=\frac{\varepsilon_{E P} \varepsilon_{0}}{\sigma_{d c}}$

And then, below the presence of EP, the low frequency dielectric constant is $\varepsilon_{E P}=M \varepsilon_{\infty}$ and therefore the parameter $M$ will be related with the relaxation times $\tau$ and $\tau_{\mathrm{EP}}$ as $M=$ $\tau_{E P} / \tau$.

When $\sigma_{\mathrm{dc}}$ is lower than $10^{-5} \mathrm{~S} \mathrm{~cm}^{-1}$ and frequency of the maximum peak in loss tangent is bigger than $1000 \mathrm{~Hz}$, then the loss tangent obtained from Eq. (2) where the real and imaginary parts have been previously separated, can be expressed as

$\tan \delta=\frac{\omega \tau_{E P}}{1+\frac{\left(\omega \tau_{E P}\right)^{2}}{M}}$

In the present study it shall be consider the dielectric measurements on films of the composite polymers (semi-IPN + deep eutectic solvent) PIA-PETA/Inulin and PIA-BAA/Inulin containing DES at different temperatures and frequencies, where a maximum in the loss tangent $(\tan \delta)$ as a function of frequency is clearly observed at each of temperatures studied. This maximum allows obtaining fits to determine the parameters that enables the calculation of the diffusion and the concentration of the free charge density under certain considerations.

Figure 8 shows the $\tan \delta$ as a function of the frequency for PIAPETA/Inulin and PIA-BAA/Inulin, in all the range of temperatures studied. A maximum in the curves at each temperature can be observed, and they shift to high frequencies as the temperature increases. The behaviour of both samples is very similar in the range of temperatures between 25 to $55^{\circ} \mathrm{C}$.
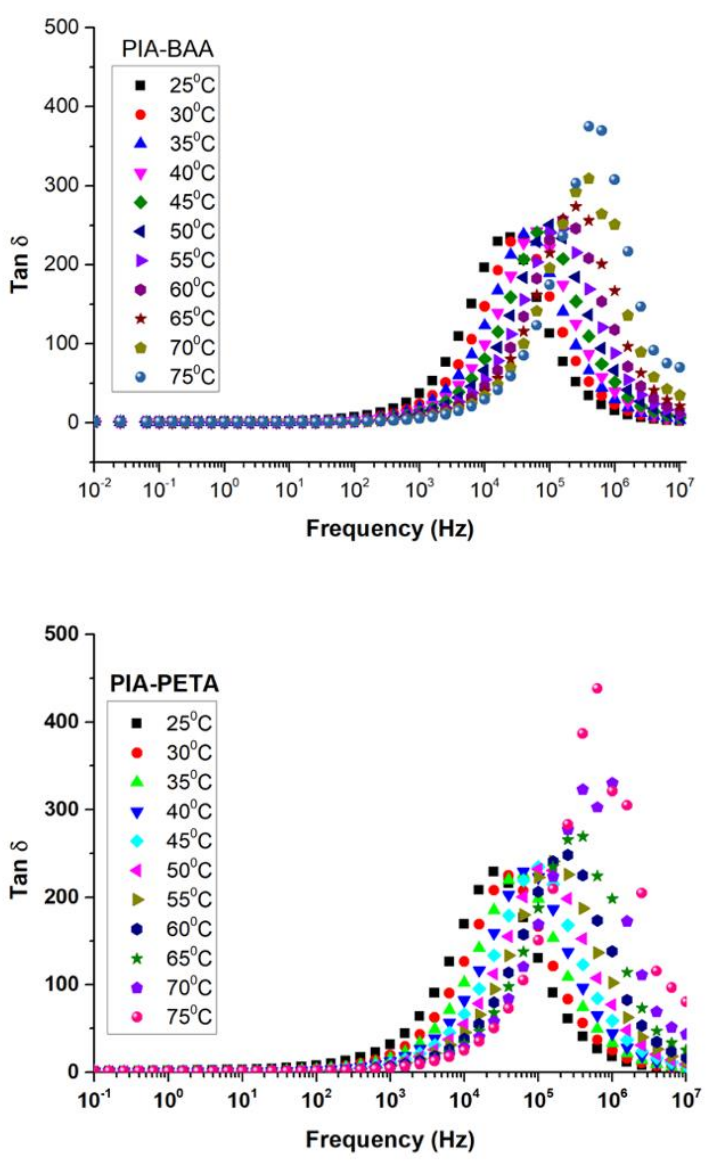

Figure 8. Tan $\delta$ versus frequency in all the range of temperatures studied for the samples PIA-BAA/Inulin (Top) and PIA-PETA/Inulin (Bottom).

Figure 9 shows the fit to the peaks showed in figure 4 for loss tangent using the Eq. (4). From the adjustment, it is determined the fitting parameters $M$ and $\tau_{E P}$. The hypothesis that the relaxation is a Debye-relaxation mean that, although interactions are present, they are not the dominant event on the transport process. It is also observed that the relaxation time $\tau_{E P}$ and the parameter $M$, depend on the temperature. In Figure 10, it can be seen the fitting for the samples PIAPETA/Inulin and PIA-BAA/Inulin at five temperatures, 25, 35, 45, 55 and $65^{\circ} \mathrm{C}$. The solid line represents the goodness of the theoretical adjustment to the experimental data. The values of these parameters are given in Table 2.

The frequency values at the peak are related with both parameters $M$ and $\tau_{E P}$ as

$\tau_{E P}=\frac{M^{\frac{1}{2}}}{\omega_{m a x}^{\tan \delta}}=\tau_{m} M^{\frac{1}{2}}$

where $\omega_{\max }^{\tan \delta}=1 / \tau_{m}$ is the reciprocal of the relaxation time corresponding to the frequency where $\tan \delta$ shows a maximum. This relaxation time is associated to the ionic conduction 
mechanism in the sample associated with the diffusivity of the free ions.
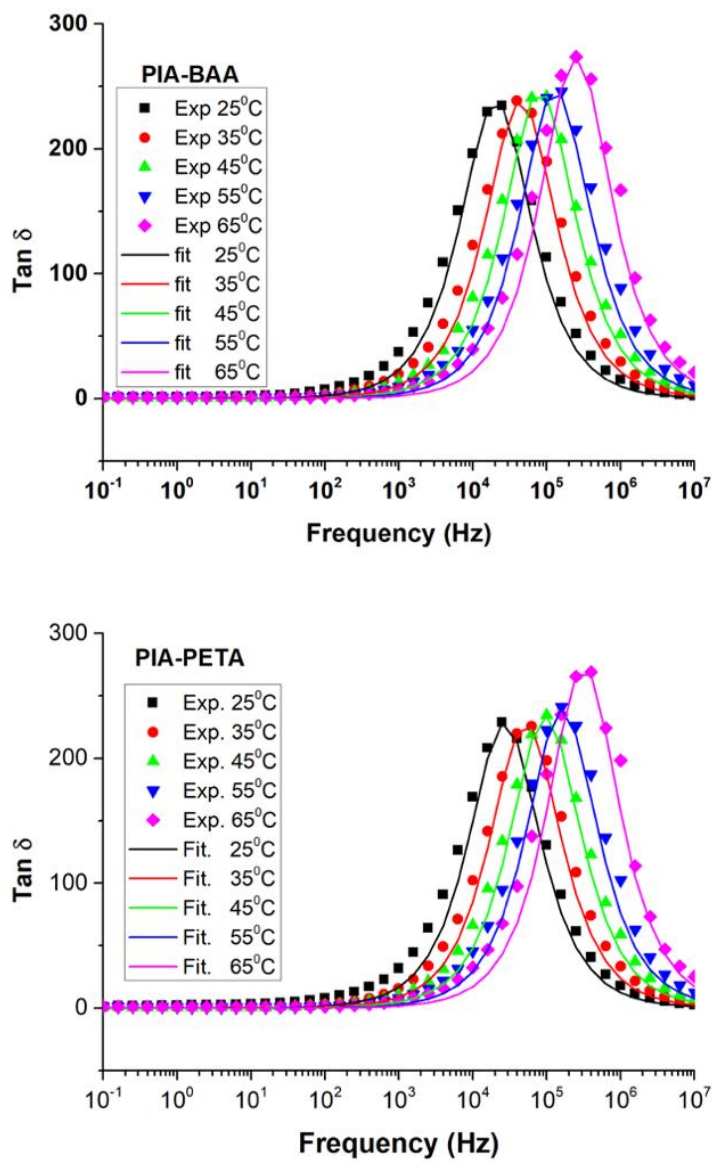

Figure 9. Tan $\delta$ versus frequency at several temperatures (25, $35,45,55$ and $65^{\circ} \mathrm{C}$ ) for the samples PIA-BAA/Inulin (Top) and PIA-PETA/Inulin (bottom). The lines represent the fit to experimental data following the Eq. (4).

Taking into account that

$\omega_{\max }^{\tan \delta}=\frac{1}{\left(\tau \tau_{E P}\right)^{\frac{1}{2}}}=\frac{1}{\tau_{m}}$

Then,

$\tau_{m}^{2}=\tau \tau_{E P} \rightarrow \tau_{m}=\tau M^{1 / 2}$

and assuming that

$L_{D}=(D \tau)^{\frac{1}{2}}$

we can estimate the effective diffusivity of mobile carriers through the samples, from equations

$D=\frac{L^{2}}{4 \tau_{E P} M}$

$D=\frac{L^{2}}{4 \tau M^{2}}$
Or

$$
D=\frac{L^{2}}{4 \tau_{m} M^{3 / 2}}
$$

For this, we fit the loss tangent curves, using the Eq. (4), to obtain $\tau_{E P}$ and $\mathrm{M}$, and then the diffusion coefficient of the semiIPN containing DES was calculated prescinding from the previous equations.

Notice that Eq. (11) is the same than that determined in Klein et al. ${ }^{14}$ but changing $\tau_{\mathrm{m}}$ by $\tau_{E P}$ and considering the Eqs. (5) and (9).

In a simple Debye relaxation, when the curves of $\tan \delta$ have a maximum, it is easy to probe that $\tau_{E P},=\tau_{m} M^{1 / 2}$. The loss tangent at the peak will be

$(\tan \delta)_{\max }=\frac{M^{\frac{1}{2}}}{2}=\sqrt{\frac{L}{8 L_{D}}}$

Such result is similar to that obtained by Sorensen and Compañ for polymeric membranes of cellulose acetate 54,60

However, in another approach, Bandara et al. ${ }^{15}$ use the relaxation time $(\tau)$ as the relaxation time corresponding to the frequency where $\tan \delta$ shows a maximum, i.e. $\tau=\tau_{m}$, in Eq (8), (see Bandara et al. ${ }^{15}$ where explicitly they stated: "the use of $\tau$ values corresponding to the maximum in the imaginary part of the electrical modulus $M^{\prime \prime}(w)$, as in reference (11) gives inappropriate values for our samples resulting in poor curve fitting. An alternative is to use the time constant corresponding to the maximum dielectric loss tangent, $\tau_{m}$. Then the relaxation time $\tau$ in eq. (7) should be actually $\tau_{m}$, where $\tau_{m}=\tau M^{1 / 2}$ ".

One possibility is that Bandara et al. ${ }^{15}$ are ambiguously using the different relaxations times $\tau, \tau_{m}$ and $\tau_{E P}$.

Table 2. Values of the fitting parameters obtained from the adjust of $\tan \delta$ using Eq.(4), along with the diffusivity (D), mobility $(\mu)$ and charge carrier density $(n)$ obtained from the fitting parameters $\left(\mathrm{M}\right.$, and $\tau_{E P}$, determined for the samples at different temperatures using the Eqs. (9), (13) and (14), respectively.

\begin{tabular}{|l|l|l|l|l|l|l|}
\hline Membrane & $\mathbf{T}\left({ }^{\circ} \mathbf{C}\right)$ & $\mathbf{M}$ & $\mathbf{t}_{\mathrm{EP}}(\mathbf{s})$ & $\mathbf{D}\left(\mathbf{c m}^{\mathbf{2}} \mathbf{s}-\mathbf{1}\right)$ & $\boldsymbol{\mu}\left(\mathbf{m}^{\left.\mathbf{2} \mathbf{V}^{-1} \mathbf{s}^{-1}\right)}\right.$ & $\mathbf{n}\left(\mathbf{m}^{-3}\right)$ \\
\hline \multirow{5}{*}{ PIA-BAA/Inulin } & 25 & 230000 & 0.0037 & $1.2 \times 10^{-5}$ & $4.6 \times 10^{-8}$ & $4.5 \times 10^{24}$ \\
\cline { 2 - 7 } & 35 & 230000 & 0.0017 & $2.6 \times 10^{-5}$ & $9.6 \times 10^{-8}$ & $4.3 \times 10^{24}$ \\
\cline { 2 - 7 } & 45 & 245000 & 0.00097 & $4.2 \times 10^{-5}$ & $1.5 \times 10^{-7}$ & $4.7 \times 10^{24}$ \\
\cline { 2 - 7 } & 55 & 245000 & 0.0006 & $6.8 \times 10^{-5}$ & $2.4 \times 10^{-7}$ & $4.8 \times 10^{24}$ \\
\cline { 2 - 7 } & 65 & 300000 & 0.00035 & $9.5 \times 10^{-5}$ & $3.3 \times 10^{-7}$ & $5.3 \times 10^{24}$ \\
\hline \multirow{5}{*}{ PIA-PETA/Inulin } & 25 & 210000 & 0.0027 & $1.8 \times 10^{-5}$ & $6.9 \times 10^{-8}$ & $3.8 \times 10^{24}$ \\
\cline { 2 - 7 } & 35 & 210000 & 0.0014 & $3.4 \times 10^{-5}$ & $1.3 \times 10^{-7}$ & $3.8 \times 10^{24}$ \\
\cline { 2 - 7 } & 45 & 220000 & 0.00077 & $5.9 \times 10^{-5}$ & $2.2 \times 10^{-7}$ & $3.8 \times 10^{24}$ \\
\cline { 2 - 7 } & 55 & 230000 & 0.00047 & $9.3 \times 10^{-5}$ & $3.3 \times 10^{-7}$ & $4.0 \times 10^{24}$ \\
\cline { 2 - 7 } & 65 & 300000 & 0.00028 & $12.1 \times 10^{-5}$ & $4.2 \times 10^{-7}$ & $4.8 \times 10^{24}$ \\
\hline
\end{tabular}


Using $\tau_{m}=\tau_{E P} M^{1 / 2}$ and taking into account the Eq. (12), $L_{D}=$ $\left(D \frac{\tau_{m}}{\sqrt{M}}\right)^{1 / 2}$ any of Eqs. (9-11) can be obtained for the diffusion coefficient.

Considering that only one type of ions is mobile through the polymer conductor the mobility can be determined from the diffusivity taking into account the Nernst-Einstein relation as

$\mu=\frac{q L^{2}}{4 \tau_{m} M^{3 / 2} k_{B} T}$

Where $\mathrm{k}_{\mathrm{B}}$ is the Boltzmann constant and $\mathrm{T}$ the absolute temperature. Finally, the mobile ion concentration was calculated from the dc-conductivity and the mobility as

$n=\frac{\sigma_{d c}}{q \mu}=\frac{4 \sigma_{d c} \tau_{m} M^{3 / 2} k_{B} T}{q^{2} L^{2}}$

Where $q$ is the charge of a monovalent cation.

Notice that Eq. (13) is different from that in Bandara et al. ${ }^{15}$, which they used as new proposed method, where an erroneous expression was determined $\mu=\frac{q L^{2}}{4 \tau_{m} M^{2} k_{B} T}$ (see Eq. 17 in Bandara et al. $)^{15}$ to calculate the mobility of the polymer electrolytes (PEO) ${ }_{68} \mathrm{Pr}_{4} \mathrm{~N}^{+} \mathrm{I}^{-} / \mathrm{I}_{2}$ at different temperatures.
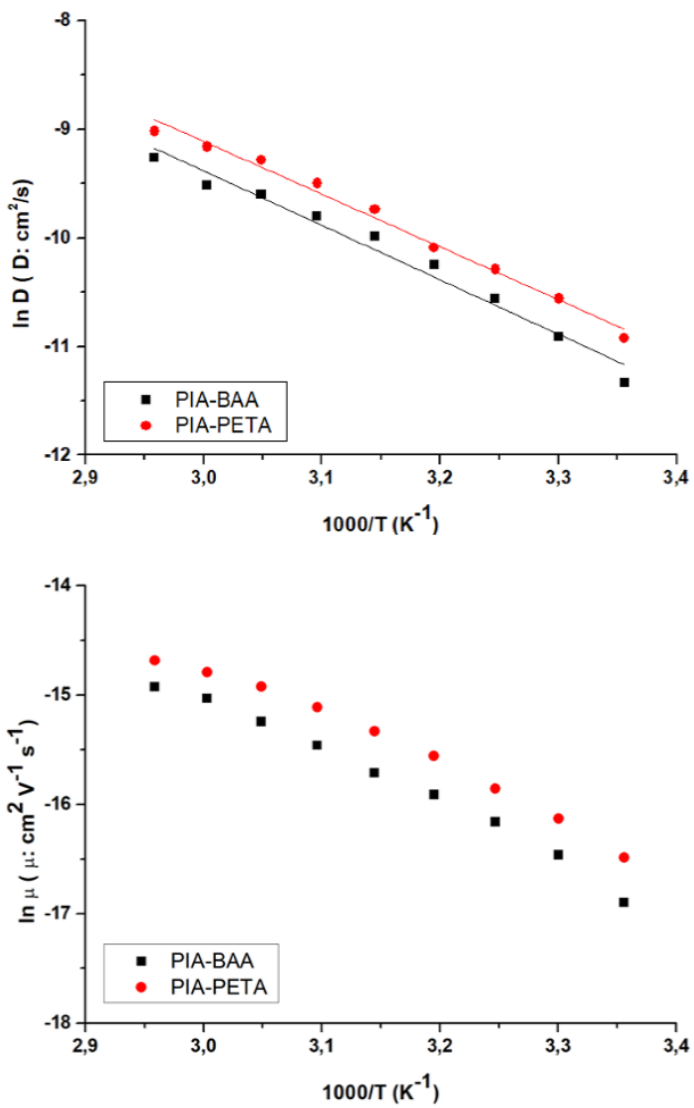

Figure 10. Temperature dependence of diffusivity (Top) and mobility (Bottom) for the ions in the semi-IPNs PIA-BAA/Inulin and PIA-PETA/Inulin.
The curves shown in Figure 8, display peaks corresponding to the maxima in $\tan \delta$ (Eq. (4)) and are associated with the plateau of the Bode diagrams showed in Figure 5 whose value represent the dc-conductivity. Their corresponding values in frequency are related with the parameters $M$ and $\tau_{E P}$ as in Eq. (5). The values obtained from the fitting peaks have been used to calculated diffusivity $D$ using Eq. (9) and Eqs. (13) and (14) to obtain the mobility and charge density, respectively. The results obtained are summarized in Table 2.

Figure 10 shows the variation of diffusivity and mobility as a function of the temperature. A close inspection of these figures shows a similar effect on diffusivity and mobility to that observed in conductivity (see Figure 7). The diffusivity rises as the temperature increases for both samples. From the slopes of the straight lines the activation energy associated to the diffusivity is calculated. The values obtained follow the trends $E_{\text {act }}(\mathrm{PIA}-\mathrm{BAA} /$ inulin $)=43 \pm 3 \mathrm{~kJ} \mathrm{~mol}^{-1}>\mathrm{E}_{\text {act }}(\mathrm{PIA}-\mathrm{PETA} /$ inulin $)=$ $40.5 \pm 2.0 \mathrm{~kJ} \mathrm{~mol}^{-1}$.

From Figure $\mathbf{1 0}$ it is observed that diffusivity and mobility exhibit the same behaviour in all the range of temperatures and both samples present the same pattern. This can be due that the ionic carriers are practically constant in all the range of temperatures and the values of the real part of the conductivity have quite similar values for the two samples. These results are surprising; in the PIA-BBA semi-IPN higher ionic migration was expected due to its lower crosslinking density as observed in the swelling experiments. Lower crosslinking density present more interconnected paths in comparison with PIA-PETA/Inulin semiIPN, which presented a higher degree of crosslinked structure and consequently a decreased the ionic migration was expected.

Altogether, the analyses of semi-IPNs containing DES as nonaqueous electrolytes have revealed that choline cations in the DES glyceline entrapped experience looser transient confinement than the HBD glycerol, despite the larger size of choline compared with glycerol. Similar results have been observed when the diffusion dynamics was analyzed on the subnanometer length scale, where the diffusive motions are larger for chloride than glycerol. ${ }^{61}$ Such behavior can be due to glycerol forming extensive hydrogen bonds with other glycerol molecules and $\mathrm{Cl}^{-}$anions, which can act as a bridge linking glycerol molecules and choline cations. It is proposed that the glycerol-glycerol hydrogen bonding correlations which are present in pure liquid glycerol is also conserved in the $\mathrm{ChCl}$-Glycerol, although the number of interactions of glycerol-glycerol hydrogen bonds may be slightly diminished or countervailed by additional interactions with chloride anions. It is known that long-range ionic diffusivity is important for the ionic transport through bulk polymers and membranes, because the ionic mobility play an important role in the chemical structure. Taking into account that glycerol-glycerol hydrogen bonding interaction are continuously presents in pure liquid glycerol we can hope that they present in our samples and also in bulk DESs involving confinement on the nanometer length scale also will be significant. The higher conductivity and diffusivity observed in PIA-PETA samples in comparison with PIA-BAA samples could be related with the long-translational diffusion, because the diffusive dynamics are dominated by the localized motions that are not strongly affected by the confinement of the DES electrolyte within the polymeric network. 
Investigations carried out in glycerol hydrogen bonding networks in Gly-ChCl DES in liquid state by neutron diffraction have conclude that rich composition in glycerol produce a homogeneous chemical structure formed by hydrogen bonded network incorporating glycerol, chloride anions and choline cations.

This analysis provides that DES-based ion gel provides a good ionic conductivity which could be an excellent material to develop of DES for supercapacitors and other electronic materials.

\section{Experimental}

\section{Materials}

All chemical was purchased from Sigma-Aldrich and was used without any further purification. Choline chloride $(\mathrm{ChCl}, \geq 98 \%)$, inulin from dahlia tubers $\left(\mathrm{M}_{\mathrm{r}} \sim 5000\right)$, citric acid ( $\left.\geq 99 \%\right)$, ethylene glycol dimethacrylate (EGDMA, $\geq 98 \%$ ), ammonium persulfate (APS, $\geq 98 \%$ ), pentaerythritol triacrylate (PETA, Sigma-Aldrich, technical grade), N, $\mathrm{N}^{\prime}$-methylenebis(acrylamide) (BAA, 99\%), glycerol (Gly, $\geq 99.5 \%$ ) itaconic acid (IA, $\geq 99 \%$ ), acrylic acid (>99\%), methacrylic acid (>99\%).

\section{Semi-IPNs preparation}

Free-radical polymerization of IA forming DES with $\mathrm{ChCl}$ (1:1 molar ratio) in bulk was carried out following the protocol reported by Bednarz et al. $\mathrm{ChCl}$ was oven-dried at $90^{\circ} \mathrm{C}$ to remove moisture. Gly-ChCl DES was prepared by mixing both components in 2:1 molar ratio, respectively, and heated at $60^{\circ} \mathrm{C}$ until a transparent and homogenous liquid was formed, same for actemide-ChCl DES. For the free-radical polymerization of itaconic acid DES monomer in solution, $1.78 \mathrm{~g}$ of IA-ChCl DES (6.6 mmol: $6.6 \mathrm{mmol}$ ) was mixed with $1.33 \mathrm{~g}$ of $\mathrm{Gly}-\mathrm{ChCl}$ DES (7.1 mmol: $3.55 \mathrm{mmol}$ ), the crosslinkers (PETA or BAA, 0.33 $\mathrm{mmol}$ ) were added, and the mixture was subjected to freeradical polymerization upon addition of APS dissolved in a minor amount of water $0.456 \mathrm{~g}$ of APS $(0.3 \mathrm{mmol}) / 0.7 \mathrm{~mL}$ water $)$.

The polymerization was carried out in closed vials at $60^{\circ} \mathrm{C}$ for 24 hours. Upon polymerization, the resulting semi-IPNs were washed thoroughly with water to remove the unreacted IA and inert DESs components, $\mathrm{ChCl}$ and Gly. Control experiments were carried without inulin with all the other conditions maintained. Also, as comparison for swelling experiments, IA was replaced by acrylic acid (acrylic acid-ChCl DES, 1.6:1 molar ratio respectively) or methacrylic acid (methacrylic acid-ChCI DES, 2:1 molar ratio respectively). For the swelling experiments, dried samples of the semi-IPN and crosslinked PIA and polyacrylic and methacrylic acid were immersed in buffers $(\mathrm{pH} \mathrm{4,7}$ and 10) at room temperature and the weight of swollen sample was recorded at the determined times. Chemical characterization of the polymeric materials was carried out by Fourier Transform Infrared (FTIR) spectroscopy. FTIR spectra of the samples were collected on an IR spectrophotometer (Perkin Elmer, Spectrum Two) using ATR (Attenuated Total Reflectance) in the range of 4000 to $600 \mathrm{~cm}^{-1}$ with a $4 \mathrm{~cm}^{-1}$ resolution, and with accumulation of 16 scans at room temperature. Samples were also characterized by solid-state Cross-Polarization Magic Angle Spinning Nuclear Magnetic Resonance, CP/MAS ${ }^{13} \mathrm{C}$ NMR. The analysis was done on a Bruker $\mathrm{AV}$ operating at $1 \mathrm{H}$ frequency of $400 \mathrm{MHz}$.

\section{Cell culture}

Human fibroblast HFF1 cell line (ATTC SCRC-1041) and murine macrophages RAW 264.7 (TIB-71), were purchased from the American Type Culture Collection. Both cell lines were cultured in Dubelcco's Modified Eagle's Medium (DMEM) (SigmaAldrich, St. Louis, MO, USA), supplemented with $1 \%$ antibiotic/antimicotic (Streptomycin/Penicilin, Blowest, USA), 2gr/L sodium bicarbonate (Sigma Aldrich, MO, USA) and 1\% LGlutamine (Biowest, USA). Cell culture media was additionally supplemented with $15 \%$ Fetal Bovine Serum (FBS, Biowest, USA) for for HFF1 cells and 10\% FBS for RAW 264.7 macrophages. Cells were cultivated at $37{ }^{\circ} \mathrm{C}$ and $5 \% \mathrm{CO}$. Once cells reached a $70 \%$ confluence in plate they were detached with trypsin-EDTA solution (Sigma-Aldrich, MO, USA). Murine macrophages were detached with a scrapper.

\section{Proliferation assay}

Prior to assess the proliferation assay, inulin material was sterilized by UV exposure and hydrated with $500 \mu \mathrm{L}$ of DMEM medium for $24 \mathrm{~h}$, cell media was replaced every $6 \mathrm{hrs}$. Then, HFF1 fibroblast cells were seeded on the top of the materials at a density of $1.25 \times 10^{4}$ cells per well in a 96-well plate and incubated for $24 \mathrm{~h}$ at $37{ }^{\circ} \mathrm{C}$ and $5 \% \mathrm{CO}_{2}$. After this, cell media was removed, and materials were taken and placed in a new well together with $400 \mu \mathrm{L}$ of DMEM media and incubated for 48 h. Cell proliferation was measured by the reduction of resazurin to resorufin by mitochondrial enzymes. After this, DMEM media was removed and cells were washed twice with PBS. Then 280 $\mu \mathrm{L}$ of DMEM media plus $20 \mu \mathrm{L}$ of resazurin at $700 \mu \mathrm{M}$ (Invitrogen, USA) were added to each well and incubated it for $4 \mathrm{~h}$ at $37{ }^{\circ} \mathrm{C}$ and $5 \% \mathrm{CO}_{2}$. Then $200 \mu \mathrm{L}$ of colored supernatant was taken and read at $570 \mathrm{~nm}$ and $600 \mathrm{~nm}$ wavelength. Proliferation of HFF1 fibroblasts was calculated according to the following Equation and a cell proliferation curve.

Resazurin reduction $(\%)=\left[\left(\mathrm{E}_{\mathrm{oxi}} 600 * \mathrm{~A}_{570}-\right.\right.$ $\left.E_{\text {ox }}\left[570 * A_{600}\right) /\left(E_{\text {red }} 570 * C_{600}-E_{\text {red }} 600 * C_{570}\right)\right] * 100$

\section{Where:}

Exi600: Molar extinction coefficient (E) of oxidized resazurin at $600 \mathrm{~nm}=117,216$

$A_{570}$ : Absorbance of test wells at $570 \mathrm{~nm}$

$E_{\text {oxi }} 570$ : $E$ of oxidized resazurin reagent at $570 \mathrm{~nm}=80,586$

$A_{600}:$ Absorbance of the sample at $600 \mathrm{~nm}$

$\mathrm{E}_{\text {red } 570:} \mathrm{E}$ of reduced resazurin at $570 \mathrm{~nm}=155,677$

$\mathrm{C}_{600}$ : Absorbance of negative control well (media, resazurin, no cells) at $600 \mathrm{~nm}$

$E_{\text {red }} 600$ : $E$ of reduced resazurin at $600 \mathrm{~nm}=14,652$

$C_{570}$ : Absorbance of negative control well (media, resazurin, no cells) at $570 \mathrm{~nm}$

\section{Nitrite production by murine RAW 264.7 macrophages}


Murine macrophages RAW 264.7 (TIB-71) were seeded at a density of $1 \times 10^{5}$ cells per well in a 96 well plate. Cells were incubated for $24 \mathrm{~h}$ at $37^{\circ} \mathrm{C}$ and $5 \% \mathrm{CO}_{2}$. After this, media was removed, and cells were rinsed with PBS 1x. Semi-IPNs previously irradiated and hydrated as described above were placed on top of the macrophages. Supplemented DMEM media without phenol-red was added to each well and cells were incubated together with inulin material for $24 \mathrm{~h}$ at $37^{\circ} \mathrm{C}$ and $5 \% \mathrm{CO}_{2}$. Then $20 \mu \mathrm{L}$ of media from each well were taken and placed in a well of a 96-well plate. Afterwards, $80 \mu \mathrm{L}$ of 5 $\mathrm{mM}$ sodium nitroprusside were added to the cell media and incubated in darkness at $37^{\circ} \mathrm{C}$ and $5 \% \mathrm{CO}_{2}$ for $1 \mathrm{~h}$. Then, $100 \mu \mathrm{L}$ of Griess reagent solution at (concentration) were mixed with the sample and incubated it at $25^{\circ} \mathrm{C}$ for $15 \mathrm{~min}$. Finally, the plate was read at $540 \mathrm{~nm}$. Absorbance values of samples were compared with a standard curve using different concentrations (1.67 to $100 \mu \mathrm{M}$ of sodium nitrite) as a reference reagent for nitrite production.

\section{Statistical analysis}

All the experiments were performed thrice with internal triplicates. Results were plotted in GraphPad Prism version 6.0 software and represent the average of data \pm standard deviation. Differences were analyzed with a one-way ANOVA test and the Student-Newman-Keuls multiple comparison test. The statistical significance was defined as $p<0.05$ analysis and a posteriori analysis were done.

\section{Conductivity measurements}

The conductivity in the transverse direction was measured by electrochemical impedance spectroscopy (EIS). The measurements were carried out on composite membranes at several temperatures in the range $25-75^{\circ} \mathrm{C}$ in steps of 5ㄷ and a frequency window from $0.01 \mathrm{~Hz}$ to $10 \mathrm{MHz}$. The experiments were performed with $100 \mathrm{mV}$ amplitude, using a Novocontrol broadband dielectric spectrometer (Hundsangen, Germany) integrated by a SR 830 lock-in amplifier with an Alpha dielectric interface. Prior to the measurements the sample thickness was measured by a micrometer, observing that in all samples the thickness did not appreciably vary less than $5 \%$. being the thickness of PIA-BAA and PIA-PETA equal to $202 \pm 5$ and $199 \pm 4$ ] $\mathrm{m}$, respectively. Samples of $20 \mathrm{~mm}$ diameter was sandwiched between two gold circular electrodes coupled to the impedance spectrometer. For this the temperature was two times firstly and gradually raised and lowered from ambient temperature to $75^{\circ} \mathrm{C}$ in steps of 5 ㅇ. At the second cycle of temperature scan, the dielectric spectra were collected in each step from $25^{\circ} \mathrm{C}$ to $75^{\circ} \mathrm{C}$. This was performed to ensure the measurements reproducibility. During the conductivity measurements, the temperature was kept constant or changed stepwise in all the range of temperatures controlled by a nitrogen jet (QUATRO from Novocontrol) with a temperature error of $0.1^{\circ} \mathrm{C}$, during every single sweep in frequency.

\section{Conclusions}

The conclusions section should come in this section at the end of the article, before the acknowledgements.

\section{Conflicts of interest}

There are no conflicts to declare.

\section{Acknowledgements}

The acknowledgements come at the end of an article after the conclusions and before the notes and references.

\section{Notes and references}

₹ Footnotes relating to the main text should appear here. These might include comments relevant to but not central to the matter under discussion, limited experimental and spectral data, and crystallographic data.

$\S$

$\S \S$

1

2
J. Le Bideau, L. Viau and A. Vioux, Chem. Soc. Rev., 2011, 40, 907-925.

P. Chakraborty, S. Das and A. K. Nandi, Prog. Polym. Sci., 2019, 88, 189-219.

A. P. Abbott, T. J. Bell, S. Handa and B. Stoddart, Green Chem., 2005, 7, 705-707.

A. P. Abbott, G. Capper, D. L. Davies, R. K. Rasheed and V. Tambyrajah, Chem. Commun., 2003, 9, 70-71.

E. L. Smith, A. P. Abbott and K. S. Ryder, Chem. Rev., 2014, 114, 11060-11082.

J. D. Mota-Morales, M. C. Gutiérrez, I. C. Sanchez, G. LunaBárcenas and F. Del Monte, Chem. Commun., 2011, 47, 5328-5330.

R. Li, T. Fan, G. Chen, K. Zhang, B. Su, J. Tian and M. He, Chem. Mater., 2020, 32, 874-881.

L. Ren'Ai, K. Zhang, G. Chen, B. Su, J. Tian, M. He and F. Lu, Chem. Commun., 2018, 54, 2304-2307.

R. Li, G. Chen, M. He, J. Tian and B. Su, J. Mater. Chem. C, 2017, 5, 8475-8481.

C. Mukesh, R. Gupta, D. N. Srivastava, S. K. Nataraj and K. Prasad, RSC Adv., 2016, 6, 28586-28592.

H. Qin and M. J. Panzer, ChemElectroChem, 2017, 4, 25562562.

B. Imre, L. García, D. Puglia and F. Vilaplana, Carbohydr. Polym., 2019, 209, 20-37.

M. F. Moradali and B. H. A. Rehm, Nat. Rev. Microbiol., 2020, $18,195-210$

R. J. Klein, S. Zhang, S. Dou, B. H. Jones, R. H. Colby and J. Runt, J. Chem. Phys., 2006, 124, 144903.

T. M. W. J. Bandara, M. A. K. L. Dissanayake, I. Albinsson and B. E. Mellander, Solid State Ionics, 2011, 189, 63-68. X. Qi, M. Watanabe, T. M. Aida and R. L. Smith, Green Chem., 2010, 12, 1855-1860.

S. Hu, Z. Zhang, J. Song, Y. Zhou and B. Han, Green Chem., 2009, 11, 1746-1749.

S. Hu, Z. Zhang, Y. Zhou, J. Song, H. Fan and B. Han, Green Chem., 2009, 11, 873-877. 
19 M. Zuo, K. Le, Z. Li, Y. Jiang, X. Zeng, X. Tang, Y. Sun and L. Lin, Ind. Crops Prod., 2017, 99, 1-6.

20 Z. Maugeri and P. Domínguez De María, RSC Adv., 2012, 2, 421-425.

L. Sapir, C. B. Stanley and D. Harries, J. Phys. Chem. A, 2016, 120, 3253-3259.

R. Stefanovic, G. B. Webber and A. J. Page, J. Mol. Liq., 2019, 279, 584-593.

Z. Chen, S. McDonald, P. FitzGerald, G. G. Warr and R. Atkin, J. Colloid Interface Sci., 2017, 506, 486-492.

M. Zdanowicz, K. Wilpiszewska and T. Spychaj, Carbohydr. Polym., 2018, 200, 361-380.

D. G. Ramírez-Wong, M. Ramírez-Cardona, R. J. SánchezLeija, A. Rugerio, R. A. Mauricio-Sánchez, M. A. HernándezLandaverde, A. Carranza, J. A. Pojman, A. M. Garay-Tapia,

E. Prokhorov, J. D. Mota-Morales and G. Luna-Bárcenas, Green Chem., 2016, 18, 4303-4311. Y. Kim, M. N. Faqih and S. S. Wang, Carbohydr. Polym., 2001, 46, 135-145.

27 J. D. Mota-Morales, M. C. Gutiérrez, M. L. Ferrer, I. C. Sanchez, E. A. Elizalde-Peña, J. A. Pojman, F. Del Monte and G. Luna-Bárcenas, J. Polym. Sci. Part A Polym. Chem., 2013, 51, 1767-1773. S. Bednarz, M. Fluder, M. Galica, D. Bogdal and I. Maciejaszek, J. Appl. Polym. Sci., 2014, 131, 40608. S. Bednarz, G. Kowalski and R. Konefał, Eur. Polym. J., 2019, 115, 30-36. R. J. Sánchez-Leija, J. R. Torres-Lubián, A. Reséndiz-Rubio, G. Luna-Bárcenas and J. D. Mota-Morales, RSC Adv., 2016, 6, 13072-13079. F. Castelli, M. G. Sarpietro, D. Micieli, S. Ottimo, G. Pitarresi, G. Tripodo, B. Carlisi and G. Giammona, Eur. J. Pharm. Sci., 2008, 35, 76-85. K. Izawa, K. Akiyama, H. Abe, Y. Togashi and T. Hasegawa, Bioorganic Med. Chem., 2013, 21, 2895-2902. G. Pitarresi, D. Triolo, M. Giorgi, C. Fiorica, F. Calascibetta and G. Giammona, Macromol. Biosci., 2012, 12, 770-778. C. V. Stevens, A. Meriggi and K. Booten, Biomacromolecules, 2001, 2, 1-16. W. Chen, X. Bai, Z. Xue, H. Mou, J. Chen, Z. Liu and T. Mu, New J. Chem., 2019, 43, 8804-8810. W. S. Ahmed Rahma, F. S. Mjalli, T. Al-Wahaibi and A. A. AlHashmi, Chem. Eng. Res. Des., 2017, 120, 271-283. K. Mielczarek, M. Łabanowska, M. Kurdziel, R. Konefał, H. Beneš, S. Bujok, G. Kowalski and S. Bednarz, Macromol. Rapid Commun., 2020, 41, 1900611. F. Afinjuomo, T. G. Barclay, Y. Song, A. Parikh, N. Petrovsky and S. Garg, React. Funct. Polym., 2019, 134, 104-111. R. H. Atalla, D. L. Vanderhart and Hackney, Int. J. Biol. Macromol., 1994, 16, 215-218. 2005, 46, 7066-7071. R. J. Sánchez-Leija, J. A. Pojman, G. Luna-Bárcenas and J. D. Mota-Morales, J. Mater. Chem. B, 2014, 2, 7495-7501.

M. Hamcerencu, J. Desbrieres, M. Popa and G. Riess, Carbohydr. Polym., 2012, 89, 438-447.
3233.

M. Shoaib, A. Shehzad, M. Omar, A. Rakha, H. Raza, H. R. Sharif, A. Shakeel, A. Ansari and S. Niazi, Carbohydr.

Polym., 2016, 147, 444-454.

M. Zhong, Q. F. Tang, Y. W. Zhu, X. Y. Chen and Z. J. Zhang, J. Power Sources, 2020, 452, 227847.

F. Zhen, L. Percevault, L. Paquin, E. Limanton, C. Lagrost and P. Hapiot, J. Phys. Chem. B, 2020, 124, 1025-1032. A. M. Navarro-Suárez and P. Johansson, J. Electrochem. Soc., 2020, 167, 070511.

Z. Xue, W. Zhao and T. Mu, Deep Eutectic Solventes: Synthesis, Properties, and Applications, ed. J. R. Diego and G. Gabriela, Wiley-VCH Verlag GmbH \& Co. KGaA, Weinheim Germany, First edition, 2020, Electrochemistry, 335-360.

S. Hong, Y. Yuan, C. Liu, W. Chen, L. Chen, H. Lian and H. Liimatainen, J. Mater. Chem. C, 2020, 8, 550-560.

A. P. Abbott, G. Capper and S. Gray, ChemPhysChem, 2006, 7, 803-806.

V. A. Kusuma, M. K. Macala, J. Liu, A. M. Marti, R. J. Hirsch, L. J. Hill and D. Hopkinson, J. Memb. Sci., 2018, 545, 292300.

L. Balo, Shalu, H. Gupta, V. Kumar Singh and R. Kumar Singh, Electrochim. Acta, 2017, 230, 123-131.

M. Zhu, L. Yu, S. He, H. Hong, J. Liu, L. Gan and M. Long, ACS Appl. Energy Mater., 2019, 2, 5992-6001.

T. S. Sarensen, V. Cornpafib and R. Diaz-calleja, J. Chem. Soc. Faraday Trans.,1996, 92, 1947-1957.

R. Coelho, J. Non. Cryst. Solids, 1991, 131, 1136-1139.

R. Coelho, R. D. Physique and E. Supérieure, Rev. Phys. Appl., 1983, 18, 137-146.

J. R. MacDonald, L. R. Evangelista, E. K. Lenzi and G. Barbero, J. Phys. Chem. C, 2011, 115, 7648-7655. M. Wübbenhorst and J. Van Turnhout, J. Non. Cryst. Solids, 2002, 305, 40-49.

U. H. Choi, A. Mittal, T. L. Price, H. W. Gibson, J. Runt and R. H. Colby, Macromolecules, 2013, 46, 1175-1186. T. S. Sørensen and V. Compañ, J. Chem. Soc. Faraday Trans., 1995, 91, 4235-4250.

D. V. Wagle, G. A. Baker and E. Mamontov, J. Phys. Chem. Lett., 2015, 6, 2924-2928. 